\title{
BIOENERGETICS, THE FOUNDATION OF THE FUTURE OF CANCER THERAPEUTICS A QUANTUM LEAP IN MAKING
}

\author{
Kambiz Afrasiabi
}

Department of Medicine and Pathology, UCI

Received 2012-07-17, Revised 2012-08-10; Accepted 2012-08-15

\begin{abstract}
Deep understanding of the governing laws of bioenergetics of the living organism and dissection of their aberrancies, could enable us to design new treatment strategies for a vast array of disorders including malignancy and could potentially lead to major breakthroughs and cure of these devastating disorders. The modern treatment strategies currently aim at identification of a pathway considered to be of unique significance in the growth and survival of a specific cell or tissue and its diseased counterpart and targeting that pathway in different ways, ranging from small molecule inhibitors, to monoclonal antibodies, at times conjugated with a toxin against a signal transduction element. In the last seventy years since the time nitrogen mustard was introduced into the treatment armamentarium of malignant disorders and until several years ago that targeted therapy became the main shift in our approach to such disorders and as of the year 2012, we have continued to ignore these fundamental principles and that has led to our failure in bringing them into treatment designs, even though we have become armed with more sophisticated technology of genomics, epigenomics, microRNA and their profiling in both normal and pathological conditions. Our main handicap has continued to be lack of deep understanding of the homeostasis of the normal state and our premature rush towards solving the puzzles of the abnormal state without such basic knowledge and understanding.
\end{abstract}

Keywords: Bioenergetics, Epigenome, MicroRNA, Genomics, Oncogene, BRCA1, BRCA2, P53, EGFR, Nanotube

\section{INTRODUCTION}

Bioenergetics implies that the fine balance of free energy of the cell since the time of inception of embryogenesis, is the main engine of cell division, survival, differentiation and organogenesis and however complex the biological processes seem to be, they rely on this simple and elegant principle of the second law of thermodynamics, which dictates that the increase in entropy/the decrease in free energy, is a function of time and since living organism is the most sophisticated and efficient anti entropy machinery, any quiescent/resting cell is at the peak of obtainable free energy, i.e., the lowest entropy and any dividing cell is attempting to reach that stage. Clearly different tissues use one or a series of pathways more preferably so in achieving this goal and that is what has been referred to as oncogene addiction by some, in their writings without paying attention to the entanglement of the living cell machinery matrix and its fate with this elegant law. One such example is Ras in pancreas and BRCA1 in breast. Along this line, cells that continue to divide without interruption are declaring their inability to achieve this goal, as they have reached insurmountable low free energy barriers and burdens, secondary to inappropriate decohescence and disentanglement of the fine-tuned cellular bioenergetics regulators from the second law derived matrix and that will be discussed in detail. Photosynthesis on which all forms of life depend, serves as the best example of the diffuse and elegant nature of this law, simply because it is dependent on the high energy macromolecule, namely chlorophyll, without which life would not have come into existence on the planet of earth, in the first place. By the same token significant decrease in the high free energy state of ovum following fertilization sends a shock wave through the 
cellular matrix, which triggers incessant rounds of mitosis to regain its original high free energy value and this leads to the complex and convoluted pathways of embryogenesis. The complex protein-protein interactions of the intracellular communication network, which resemble a hairball (around three hundred thousand different interactions by one estimate and probably more), represents the highest free energy allowable by the second law, in contra distinction to its diseased and malignant counterpart which has a significantly lower value, in order to correct this problem, rather than going after the intricacies of this network, a much more practical and efficient way is to calculate the hairball free energy of normal cell and compare it with that of its malignant counterpart and decipher the difference, by using the available mathematical models and design a hairball free energy gap closer/HFEGC or normalizer strategy which could be done at the epigenome, or other gene regulatory stations, such as microRNA network and this may necessitate approach to certain unfamiliar genes and gene regulatory compartments, totally unpredicted today (Hsu and Sabatini, 2008). I would like to proceed with major examples of malignant disorders, which follow a common theme and touch on these points in a practical and didactic way as we move on and by applying the methodology of identification and dissection of the dynamics of the key regulators of the bioenergetics homeostasis of normal and malignant counterparts, I would propose a new curative treatment modality, according to those parameters.

\subsection{Adenocarcinoma of Pancreas}

one of the deadliest of all malignant disorders with a case fatality rate of $85 \%$ and almost no meaningful benefit from current therapeutic modalities. K-Ras activating mutations have been identified in the vast majority of the cases and targeted therapy with FTI/farnesyl transferase inhibitors has failed, because of total lack of understanding of the of Ras role in homeostasis of the bioenergetics of the pancreatic duct cell, i.e., its pivotal role in promoting the increase in the free energy of the pancreatic ductal cell following daily physiological recurrent sinusoidal falls. In a normal pancreatic duct cell this goal is easily achieved and following activation of recurrent rounds of mitosis by activated Ras, Ras GAP/Ras GTPase activating protein terminates further cell division by converting GTP attached to Ras to GDP, which leads to detachment of Raf from Ras, which is sitting downstream of Ras and upstream of other signal transduction elements connecting the proliferative message of Ras to the nucleus of the cell; in adenocarcinoma of pancreas, activating point mutations designed by the stem cell compartment incessantly activate the downstream pathway and inability of the malignant pancreatic duct stem cell compartment to normalize the bio energetics of the cell, i.e., increase its free energy to the normal level, leads to employment of more mutations by the malignant stem cell compartment, to achieve the unachievable goal and deeper malignant events ensue. Approaching Ras pathway in the treatment of adenocarcinoma of pancreas, is a reflection of the current misperception of its role as the cause, though indeed it is only one reflection out of probably many others, of a deeply seated pathology in the cell energetics by processes that have taken the cell into irreversible deep valleys of low free energy and high entropy. Indeed Ras Gap mutation itself, which leads to constant attachment of Ras to GTP, in the absence of activating point mutations of Ras and thus leading to constant activation of that pathway, represents a lower free energy quaternary conformation, simply because after cessation of cell division and achievement of the intended increase in free energy in a normal pancreatic duct cell, that complex would convert to Ras-GDP, which represents an alignment with the higher free energy state of the cell and this cycle would resume as needed, under normal conditions, following daily physiological disturbances. Other genetic and micro- environmental etiologies of adenocarcinoma of pancreas, such as BRCA1, BRCA2, HNPCC and chronic pancreatitis, which have to do with duct branching defects, mismatch repair deficiency and increase in hypoxic niche, respectively, are acting as the evidence that pancreatic carcinogenesis could start from totally different points and pathways, albeit with one common denominator of decrease in free energy of the pancreatic duct cells. In order to cure adenocarcinoma of pancreas, we need to generate a higher free energy state in the pancreatic duct malignant stem cell compartment, matching its normal counterpart and in order to achieve this goal, we either need to generate a higher free energy hairball, which should exactly match that of the normal pancreatic duct stem cell hairball free energy, or manipulate the genetic regulatory switchboard of the malignant pancreatic duct stem cell compartment master regulator complex to match that of its normal counterpart, this could be deciphered based on the available mathematical models.

\subsection{Carcinoma of Lung}

Comprising $14 \%$ of all cancers and $29 \%$ of all cancer related mortalities, little progress has been made in screening and treatment of this devastating disease in the last several decades, again we are busy with our childish 
playing with superficial targeted therapies. EGFR is up regulated in $70 \%$ of all non-small cell carcinomas of the lung, which comprise around $80 \%$ of all lung cancer cases, p53 is mutated in $75 \%$ of small cell and $50 \%$ of non-small cell carcinomas of the lung, K-Ras is mutated in $30 \%$ of adenocarcinoma cases. All these represent the futile response of the stem cell compartment to the inappropriate decrease in free energy of the neoplastic stem cell compartment, through acceleration of mitosis in an attempt to normalize, i.e., increase the free energy of the stem cell compartment and the ensuing further buildup of ill mutations, as the previous mechanisms fail and the problem adopts deeper magnitude and gravity. The scope of the mutations in each disease example, in a way represents the thinking/repair design of the master regulator cell, namely the stem cell to resolve the problem and clearly identification of that signature would act as the main platform of our future treatment strategy and understanding of the depth of the problem, e.g., the more of the proliferative signatures that we encounter in one disease, the earlier we are in the malignant phenotype natural history and the easier it would be to design a curative treatment modality and the more diversified the spectrum of the malignant cell signature, the more convoluted and difficult it becomes to treat that malignancy. I propose two major approaches to treatment of carcinoma of lung, one that is based on the general hairball energetics aberrancies and the other which is groove based and the depth and location of the groove is best defined as specific signature of the mutation profile of that particular malignancy, e.g., an adenocarcinoma of lung with EGFR exon 18 mutation, p53 mutation and bcl2 upregulation, is telling us that the stem cell compartment has decided to paralyze the programmed cell death machinery and continue with uninterrupted rounds of mitosis in order to create a higher (appropriate for that stage of that cell) free energy, the spectrum of which could spread from epigenome, all the way to the cytoplasmic hairball (Vousden and Ryan, 2009). It is incumbent upon us to identify the master regulator complex of each cell type, upon which the whole integrity and functionality of that cell type depends and the aberrancies of which, lead to the aberrancies in the free energy of the master regulator complex of that cell type, which leads to distortion of the functionality and integrity of that specific cell, which is one of the hallmarks of malignant phenotype, in case of non-small cell carcinoma of the lung I propose the genetic machinery in charge of gland and duct formation as well as branching and formation of alveoli, which comprise the identity of that organ, as the master regulator complex and in case of small cell carcinoma of lung one needs to look into the genes moderating the transition of nervous and endocrine systems, as the main players/master regulator complex. Designing the reversal of these aberrancies rather than pushing the brake on the prescriptions of the stem cell compartment (mutations), with agents such as Tarceva, Avastin and Herceptin, should become the main focus of future generation cancer therapeutics design, which is expected to change modest survival benefit to cure of these devastating diseases.

\subsection{Carcinoma of Breast}

The most common cancer in females in the USA and the second most common cancer related cause of death in females, yet other than tamoxifen, faslodex and aromatase inhibitors for hormone receptor positive breast cancers and chemotherapy+/-HER-2 targeted therapy and most recently PARP inhibitors for selected patients and with limited efficacy, on top of mastectomy or lumpectomy with or without radiation therapy, there is not much to offer to such patients (Rodon et al., 2009). Whole genome sequencing, epigenetics and micro RNA mediated targets are helping to add to the treatment options; however there is no hope for a home run in combating this disease any time soon. Again we have been following the same format in treating breast cancer and other cancers such as lung, colon and prostate, as though our treatment designers are looking over each other's shoulders.

One more time we need to revisit the fundamentals of the bio energetics in the embryogenesis, development and homeostasis of this organ, which is dependent upon major genes such as BRCA1 and BRCA2, for its branching and so sensitive to estrogens and progestational agents for growth and secretion and prolactin for milk formation. Breast tissue is anchoring on fat, which is full of stored energy/low in free energy (high in entropy) and armed with aromatase which could convert androgen to estrogen (heat generator), a second dominant element of increasing loco-regional entropy. The fact that the incidence of breast cancer in men is $1 \%$ that of women, in and of itself talks about the significance of female hormones, in the development of breast cancer, the major contribution of female hormones in generation of breast cancer is triggering of proliferation of breast duct and lobule cells, through activation of resting stem cells in these zones and taking them out of G0 and G1 and heat generation (by intra breast tissue estrogen), which leads to a decrease in free 
energy of different sub cellular compartments of breast stem cells, from epigenome, all the way to microRNA and cytoplasmic hairball, via simple heat diffusion. In addition the fact that the vast majority of females with BRCA1 and BRCA2 mutation, develop breast cancer, points toward the significance of the proper duct formation and branching modulated by these genes. In a normal breast, duct formation and branching follow the path of the highest free energy/lowest entropy, i.e., respecting the second law of thermodynamics, In contrast, in a BRCA1 and BRCA2 mutated breast, duct formation obviates this law, i.e., the configuration and topology of breast ducts carry a lower free energy/higher entropy and this higher entropy extends through all the sub compartments of the duct and lobule cells and as the bio unit/the cell is an anti-entropy machinery, the corrective mechanisms, which mostly employ proliferation, cannot catch up with this erroneously built in low free energy unit and further mutations are employed by the stem cell compartment to achieve this unachievable goal and by doing so, through time a full blown malignant phenotype arises. The cure of breast cancer resides in the calculation and formulation of the bio energetics of different sub compartments of this organ, at all levels from ductules to lobules to major ducts. One such approach is calculation of the hairball free energy of each sub compartment, another approach is direct hairball groove based approach (groove is defined as the location and configuration of master regulator complex of the cell in the hairball), similar calculations need to be done in the malignant counterparts and this would act as a foundation for generation of mathematical models, which would create the opportunity to design a complete reversal of the gap, either through epigenetic, or micro RNA modification, or direct normalization of the breast cancer hairball energetics, which might be achieved by shuttling nanotubes with pre-determined vibratory energy, modifying and balancing the energetics of the master regulator section (groove of the hairball), this could be considered the future of customized medicine, which demands a major shift in our thinking from the current rudimentary way to a deep and scientific understanding of the fundamental laws governing the bio universe and their application towards the cure of catastrophic diseases such as cancer.

\section{MATERIALS AND METHODS}

Calculation of the free energy of the hairball might prove challenging, but by using indirect measures such as inserting a nano electrode into the cytoplasm of the malignant cell and its normal counterpart and measuring the number of vibrations or fluctuations per nanosecond, or nano voltage, we could come up with representing differences in the free energy of normal and cancer cell and use those numbers in the buildup of a mathematical model, which could serve as the template for our treatment design in targeting epigenome or micro RNA compartments (Crowder et al., 2005). In essence bioenergetics is sitting at the heart of the bio system and shapes the matrix of the bio universe and decides about its fate since the time of its inception to the moment of its demise and unfortunately the current cancer therapeutic designers are totally blind to this most fundamental principle and it is only when we could appreciate its precise role that we would become able to revolutionize our approach to understanding and cure of diseases such as malignant disorders and take a quantum leap out of the dark corridors of the cancer therapeutic designs of today. The aberrancies of the energetics of the hairball of the malignant cell, in comparison with its normal counterpart, could also reflect themselves in one or a small number of grooves in the whole complex protein-protein interaction network and that could prove advantageous to the future cancer therapeutic designers, simply because it could generate a small visible tract to follow and repair. One such groove could encompass the upregulated tyrosine kinase network, which happens in the vast majority of malignancies (Mahon et al., 2000).

\section{RESULTS AND DISCUSSION}

By applying the same principles to each organ, we could come up with similar curative designs for different malignancies, for example, in case of adenocarcinoma of prostate, we need to recognize the androgen receptor shuttling protein-protein interaction network as the hairball groove, in case of neoplastic disorders of lymphocytes, their surface receptor proteins (for both B and $\mathrm{T}$ cells) and VDJ rearrangement genetic machinery of B cells, could comprise the groove (Costello and Franklin, 1994). In case of adenocarcinomas of the GI tract, the cell fate determining genetic machinery and their representative protein- protein interaction network, at the base of the crypt, could comprise the hairball groove. In case of sarcomas, the specific genetic machinery in charge of specific identity of that cell type and in case of gliomas, the genetic machinery of astrocytes that codes for niche protein network, should 
be examined critically as far as groove designation concept is concerned.

In our final design of the future of cancer therapeutics, we should also consider the loco regional topology of that specific organ and its responsible genetic machinery and protein network, handling the energetics of that particular micro environment, just as fat and aromatase of fat cells, surrounding breast tissue were discussed earlier.

\section{CONCLUSION}

The deep understanding of the fundamentals of the bio energetics, which originates from the interplay of the second law of the thermodynamics with the bio universe and the recognition of its diffuse and elegant presence at all sub cellular levels and the acquisition of their values in both normal and malignant cells, would enable us to develop mathematical models for each disease example, which could act as the platform for delivering the corrective treatment modality, to restore the normal energetics for each particular disease example, this at times might demand shuttling of nano tubes with inherent vibratory potentials, which could get attracted to the groove of the intracellular hairball (the site of the master regulator complex of that specific cell) and at other times highly calculated manipulation of the Epigenome and microRNA network, in favor of normalization of the perturbed energetics of that specific disease example (Elhissi et al., 2012). All this demands a leap out of our current illusionary views towards pathogenesis of malignant disorders and putting the dedicated positive research forces together, on an urgent basis, this would empower mankind to win one of the biggest battles against an enemy which has afflicted humanity for ages.

\section{REFERENCES}

Costello, L.C. and R.B. Franklin, 1994. Bioenergetic theory of prostate malignancy. Prostate, 25: 162166. PMID: 7520580
Crowder, K.C., M.S. Hughes, J.N. Marsh, A.M. Barbieri and R.W. Fuhrhop et al., 2005. Sonic activation of molecularly-targeted nanoparticles accelerates transmembrane lipid delivery to cancer cells through contact-mediated mechanisms: Implications for enhanced local drug delivery. Ultrasound Med. Biol., 31: 1693-1700. PMID: 16344131

Elhissi, A.M., W. Ahmed, I.U. Hassan, V.R. Dhanak and A. D'Emanuele, 2012. Carbon nanotubes in cancer therapy and drug delivery. J. Drug Deliv. PMID: 22028974

Hsu, P.P. and D. Sabatini, 2008. Cancer Cell Metabolism: Warburg and Beyond. Whitehead Institute for Biomedical Research and Massachusetts Institute of Technology Department of Biology, Cambridge, MA 02142, USA. Cell, 134: 703-707. PMID: 18775299

Mahon, F.X., M.W. Deininger, B. Schultheis, J. Chabrol and J. Reiffers et al., 2000. Selection and characterization of BCR-ABL positive cell lines with differential sensitivity to the tyrosine kinase inhibitor STI571: Diverse mechanisms of resistance. Blood, 96: 1070-1079. PMID: 10910924

Rodon, J., M. Iniesta and K. Papadopoulos, 2009. Development of PARP inhibitors in oncology. Expert Opin. Investig. Drugs, 18: 31-43. PMID: 19053880

Vousden, K.H. and K.M. Ryan, 2009. p53 and metabolism. Nat Rev. Cancer, 9: 691-700. DOI: $10.1038 /$ nrc2715 